This is the Accepted Version of the article: Haolin Zhang, Angel Lizana, Albert Van Eeckhout, Alex Turpin, Claudio lemmi, Andrés Márquez, Ignacio Moreno, Fabian A. Torres-Ruiz, Asticio Vargas, Francesc Pi, Juan Campos, "Dynamic microparticle manipulation through light structures generated by a self-calibrated Liquid Crystal on Silicon display ," Proc. SPIE 10677, Unconventional Optical Imaging, 1067720 (24 May 2018).

Available at DOI: https://doi.org/10.1117/12.2309385

(C) 2018 Society of Photo Optical Instrumentation Engineers (SPIE). One print or electronic copy may be made for personal use only. Systematic reproduction and distribution, duplication of any material in this publication for a fee or for commercial purposes, or modification of the contents of the publication are prohibited. 


\title{
Dynamic microparticle manipulation through light structures generated by a self-calibrated Liquid Crystal on Silicon display
}

\author{
Haolin Zhang, Angel Lizana, Albert Van Eeckhout, Juan Campos, Univ. Autònoma de Barcelona \\ (Spain); Alex Turpin, Caesar (Germany); Claudio Iemmi, Univ. Buenos Aires (Argentina); Andrés \\ Marquez, Univ. Alicante (Spain); Ignacio Moreno, Univ. Miguel Hernández de Elche (Spain); \\ Fabian A. Torres-Ruiz, Asticio Vargas, Univ. Frontera (Chile)
}

\begin{abstract}
This paper is devoted to investigate the application of different dynamic light structures generated by a self-calibrated Liquid Crystal on Silicon (LCoS) display for microparticle manipulation. Two major studies based on implementing different DOEs, to thoroughly characterize the LCoS display and to achieve optical-inspired particle manipulation, are proposed, respectively. On the one hand, we dynamically introduced the billet-lens configuration and the micro-lens array pattern on the LCoS display, from which the self-calibration of the studied device is implemented. In this case, both the phase-voltage relation and the surface profile were determined and optimized to the optimal performance for microparticle manipulation. On the other hand, we performed the optical manipulation of microparticles by addressing configurable three-dimensional light structures obtained from different phase driven split-lens configurations initiated by the same but optimized LCoS display. Experimental results demonstrated that, by addressing certain phase distributions on the LCoS display, the microparticle can be trapped in the light cones and manipulated by providing certain continuous split-lens configurations.
\end{abstract}

Keywords: Liquid-crystal devices, Diffractive optical element, Calibration, Optical trapping, Phase modulation, Holographic display

\section{INTRODUCTION}

The interest of microparticle manipulation through optical mechanism for the investigations in physical and biological fields has been widely discussed recently. For instance, in the physical perspective, optical microparticle manipulation provides the feasibility to fulfill atom cooling for the acquisition of atomic collision [1, 2] or the acceleration of heavy ion beams [3]; to develop atom interferometers for high-precision gravity acceleration measurement [4]; to realize novel atom mirrors [5] or to obtain a flexible hollow optical fiber [6]. In the biological perspective, optical manipulation allows to control the living cells or the biological molecules remotely [7, 8]; to manipulate chromosomes during the cell division [9], among others. To realize the microparticle manipulation, the particle is to be firstly trapped in a light generated bottle structure [10-15] which suspends the particle in the air by photophoretic force [16]. Moreover, this generated light bottle structure should be able to be spatially controlled to realize the microparticle dragging. To perform this manipulation (i.e., the optical trapping and the particle dragging), a Liquid Crsystal on Silicon (LCoS) display is introduced by being addressed different digital diffractive optical elements (DOEs) [17] to obtain the controllable bottle-shape light structure.

Liquid Crystal on Silicon (LCoS) displays are one type of spatial light modulators (SLM) sketched in the reflective configuration [18]. Specifically, an LCoS display provides the feasibility to modulate the income wavefront from the amplitude or the phase perspective [19]. As an example in the amplitude modulation perspective, LCoS displays can be introduced in ground-based telescope systems [20] to eliminate the air turbulence aberration [21] as an adaptive optical element. On the phase modulation regime, LCoS displays are extensively applied to implement the optical tweezers for particle trapping [22], to modulate the polarization to enhance the diffraction efficiency [23-25], or they are performed as the pattern generator in the real-time laser beam shaping technique to realize an optical tractor [26], etc. In the abovementioned microparticle manipulation cases, as the light bottle structures are mainly controlled by addressing DOEs, the $\mathrm{LCoS}$ display introduced is operated in the phase-only regime. However, the disproportion of the phase-voltage relation and the spatial phase inhomogeneity $[27,28]$ of the LCOS screen related with fabrication inaccuracy distort the DOE patterns generated, which influence the obtained light bottle structures for particle manipulation. Therefore, the precalibration and the optimization of the imperfect phase distribution of the LCoS are mandatory. Thanks to the capability 
of an LCoS display to self-adjust its phase property, a self-calibration of this SLM device is applicable. Numbers of LCoS self-calibration methods, both optimize the phase-voltage relation and the surface phase inhomogeneity, are proposed in literatures [27, 29]. Among them, interferometry [30, 31] or diffraction [32, 33] based methods are demonstrated suitable for the phase-voltage calibration, and the Shack-Hartmann pattern [34, 35] meets the requirement to imply the surface inhomogeneity calibration.

In this paper, the phase calibration of the LCoS display, which is subsequently used for particle manipulation, is firstly implemented. Specifically, we addressed a Billet-lens configuration, implies an interferometry system, suitable for the phase-voltage relation identification. Afterwards, the surface phase profile calibration is obtained by introducing a micro-lens array pattern. On the other hand, once the LCoS display is calibrated and optimized to its optimal condition, we carried out the microparticle manipulation by employing the same but optimized LCoS display. Under this condition, we firstly performed a light bottle structure to realize the trapping of the particle by introducing a certain split-lens configuration and a multiplexed diffractive lens pattern together on the LCoS. Later, by dynamically adjusting the DOEs driven to the LCoS for the light bottle structures, the trapped particle was spatially dragged in the optical axial direction in which a tailored tractor beam is formed.

The outline of this manuscript is described as follow. In Section 2, we described the LCoS display self-calibration methods and measured the calibration parameters (i.e., the phase-voltage curve and the surface phase profile). Afterwards, the calibration results obtained were directly implemented to the LCoS to realize the optimization. In Section 3, the conception of microparticle manipulation by utilizing different DOEs generated by the already calibrated LCoS display is demonstrated. Moreover, we experimentally presented the trapping of a microsphere (diameter of $\sim 65$ $\mu \mathrm{m})$ and the axial spatial manipulation of the trapped particle with certain dynamic split-lens configurations. In Section 4 , the main conclusion and contribution of the LCoS display provided particle manipulation are given.

\section{SELF-CALIBRATION OF THE LCOS DISPLAY}

To generate the bottle shape light structure for the efficient microparticle manipulation, certain phase distributions should be addressed to the LCoS display as above mentioned. Therefore, to utilize the LCoS display at its optimal condition, a precise phase distribution calibration should be performed. In our case, two characteristics, the phasevoltage relation as well as the surface phase inhomogeneity of the LCoS display are to be calibrated. Thanks to that the LCoS display can self-address different Diffractive Optical Elements (DOEs) to itself, the characteristics of the LCoS can be easily determined.

\section{A. LCoS display phase-voltage relation calibration}

In this section, we discussed the calibration of the LCoS phase-voltage relation by addressing a certain split-lens figure, described as the two-sectorial Billet lens [17]. The corresponding phase distribution describing this pattern as a symmetrical lens split by half with the separation distance of the two sectors as $a$ is shown in Fig. 1(b). Once this pattern is fully illuminated by the collimated light, each sector of the Billet lens focuses the light into their own focal points. Afterwards, the two focal points being propagated into the far field meet each other and an interference fringe pattern is generated. Note that the interference fringe period is determined by both the separation distance $a$ and the focal lengths $f$ of the two sectorial lenses. Under this scenario, by digitally controlling the parameters of the Billet lens (i.e., $a$ and $f$ ), the alteration of the fringe is easily achieved. More importantly, the phase-voltage relation can be retrieved from the obtained interference fringe. In our case, the phase distribution of the first half lens is set constant while we continuously add a constant phase value (related with gray level) controlled by the driven voltage to each pixel describing the second sectorial lens. Therefore, the continuous phase adjustment in the second sector leads to the lens configuration alternation and therefore inspires an interference pattern shift. We want to note that we regulate the phase values added to the second lens with respect to the gray levels ranging from 0-255. Hence, we provide a whole gray level range to fulfill the phase-voltage measurement. Finally, the interference shift, characterized as the pattern displacement which is distinguishable by the receiving CCD, is recorded and applied to calculate the phase-voltage relation [27]. 


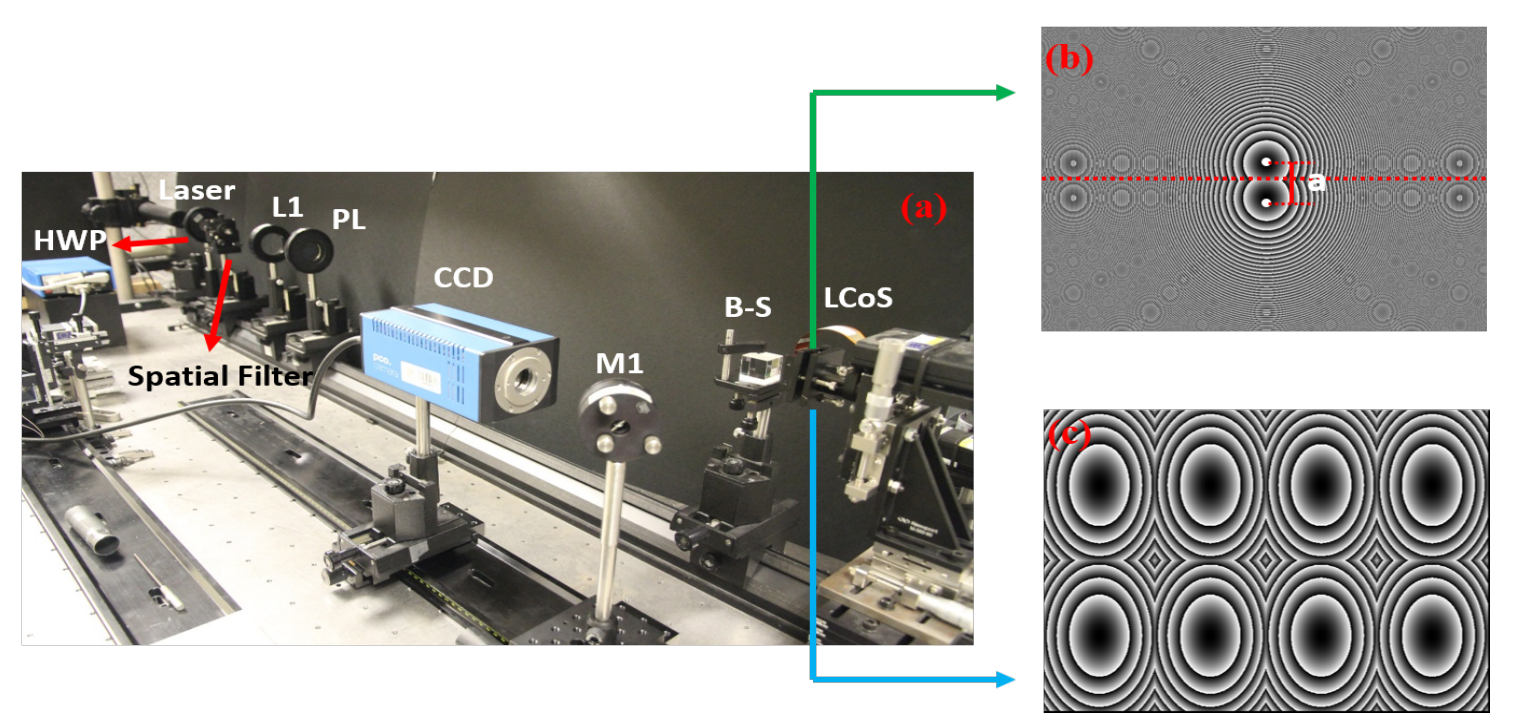

Fig. 1: Experimental implementation for the LCoS display calibration: (a) Experimental set-up for the phase-voltage curve and the surface phase profile calibration; (b) The phase distribution of the Billet-lens configuration; and (c) The phase distribution of the micro-lens array configuration.

The phase-voltage calibration is implemented as the above-explicated self-calibration method. We measured the phase-voltage curve of a PLUTO parallel aligned LCoS display distributed by HOLOEYE, which in the further discussion, is to be applied for the optical particle trapping. The LCoS display presents a resolution of $1920 \times 1080$ with the pixel size as $8 \mu \mathrm{m}$. The phase distribution on this modulator for the phase-voltage calibration is demonstrated as Fig. 1(b). To better illustrate the calibration system, Fig. 1(a) is introduced to explain the experimental layout. In the system sketch, we use a He-Ne laser (wavelength of $632.8 \mathrm{~nm}$ ) to serve as the illumination source. Afterwards, the illumination passing through the half waveplate and the linear polarizer, which is utilized to generate the linear polarized light, is filtered and collimated by the spatial Filter and the collimated lens L1. Finally, the interference pattern is obtained by the CCD with two reflections provided by the beam-splitter and the flat reflective lens M1. At this moment, the light propagating to the LCoS display, is fully linear polarized and collimated. On the LCoS display, the phase distribution of the Billet-lens configuration, described as Fig. 1(b), is addressed to the SLM with the sectorial distance as $0.4 \mathrm{~mm}$ and the focal lengths of the two sectorial lenses as $350 \mathrm{~mm}$. Meanwhile, this phase pattern is gradually altered by implying a constant gray level (related to phase value) to the lower half section, which is directly conducted by the change of the driven voltage, with a gray level step difference of 8 ranging from 0 to 255 . Hence, we obtained 33 different interference patterns on the CCD correspond to each gray level driven. The displacement of each interference pattern is extracted and the post-processing for the acquaintance of the phase-voltage relation is conducted [27]. Moreover, we conducted this measurement for each gray level period (0-255) repeatedly for one hundred times in order to study the robustness of the proposed method. The robustness is demonstrated by the calculation of the standard deviation of these one hundred time measurements. The final phase-voltage curve corresponds to the mean phase values (blue curve) with the standard deviation (represented as red bar) included is demonstrated in Fig. 2. The mean phase values range from 0 to $\sim 6.28$ radians with the gray level arrays from 0 to 255 with a strict linear tendency. What is more, the maximum error of the obtained curve is of 0.22 radian. We want to note that this behavior fits the commercial characteristic of the proposed HOLOEYE LCoS display. What is more, the standard deviation shows a strong robustness with a small error in each gray level driven. 


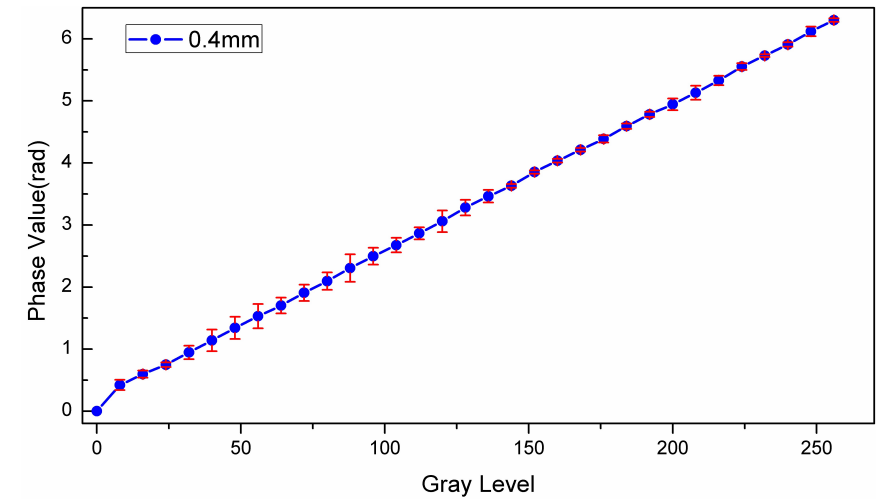

Fig. 2: Average phase value versus gray level measured by a Billet-lens configuration with the separation distance of $0.4 \mathrm{~mm}$.

\section{B. LCoS display surface phase profile characterization calibration}

The top layer of the LCoS device is a piece of cover glass which is not completely flat considering the mechanical stress imported during the fabrication. Under this scenario, the surface profile error presents an unfavorable extra phase distribution. For that reason, the surface phase profile calibration has to be considered to eliminate this unsatisfactory phase and further improve the characterization of the SLM, which is useful for the particle manipulation.

To calibrate the surface phase profile, we proposed a mirco-lens array pattern (Shack-Hartmann pattern) for their capability to fulfil the wavefront aberration measurement. Specifically, we generate the phase distribution describing a micro-lens array to the LCoS display and illuminate with a plane wavefront. Under this scenario, the focal points correlated with each micro-lens is obtained at the same focal plane if each micro-lens shares the same focal length. More importantly, the distance between each two neighboring focal points presents a same length if the input wavefront and the surface of the SLM generating the micro-lens array are strictly plane. On the contrary, an inhomogeneity distribution of the focal points on the focal plane reveals a distorted surface from flatness if the illumination is collimated. Under this scenario, by generating the same focal length micro-lens array and collect their corresponding focal point distributions with a collimated illumination, we can determine the surface profile and further, correct the surface distortion for a better microparticle manipulation.

In our case, we use the same LCoS display with the phase-voltage relation already well calibrated (Seciton 2. (A)) to firstly generate a $4 \times 2$ micro-lens configuration. The phase distribution of this micro-lens array is presented in Fig. 1(c), with each micro-lens shares a size of $400 \times 400$ pixels. Therefore, the whole dimension of the micro-lens is of $1600 \times 800$ pixels, and 8 light spots are found on the focal plane. Note that this $4 \times 2$ micro-lens pattern dimension $(1600 \times 800)$ cannot cover the whole size of the LCoS screen $(1920 \times 1080)$. What is more, the small account of the light spots (i.e., 8 focal points) restricts it to achieve a high resolution calibration. To overcome this disadvantage, we imply the displacements of the initially obtained 8 light spots on both the vertical direction and the horizontal direction of the LCoS for 8 steps with each step length as 50 pixels. This is easily feasible by digitally shifting the phase distribution of the 8 micro-lens configurations a constant distance along the LCoS display. In this case, we finally accumulate the whole intensity distribution within the CCD as a $32 \times 16$ focal light spot, with the displacement of the neighboring points as 50 pixel size (the pixel size of the LCoS display). The final light spots pattern is presented in Fig. 3. To acquire the spatial position of each spot, we introduced a $38 \times 38$ equally dispersed grid square to locate each light spot by taking into consideration the pixel matching of the LCoS display and the CCD [27]. The grid distribution can be distinguished from Fig. 3. At this moment, the spatial position of each point corresponds to the certain $38 \times 38$ grid pattern is determined, the distances of any light spots differ from the grid centers are calculated. Note that this light spot distribution is not uniformly distributed, the displacements of each spot to their grid center are different. As the differences in the displacements are related to the LCoS surface shape deviation from the strict flatness, the surface derivative in any light spot can be determined by applying the obtained displacement values using an integration algorithm [34]. Finally, the surface profile is estimated by using a cubic spline interpolation with the above-obtained derivatives. 


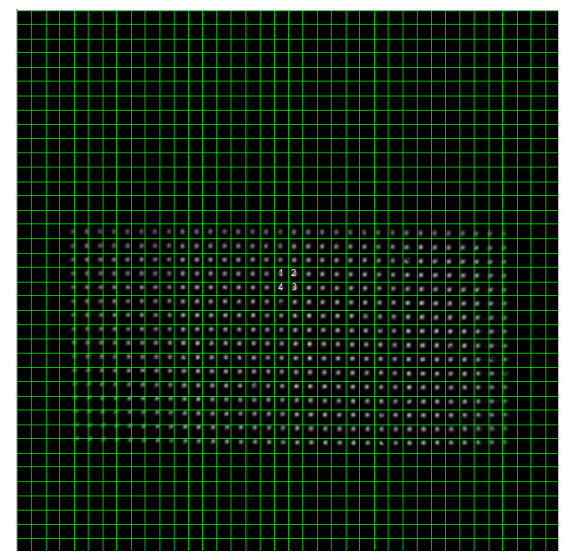

Fig. 3: Light spot distribution located in a $38 \times 38$ square grid.

The surface profile calibration is launched by using the same optical setup depicted in Fig. 1(a), but only a different phase pattern as the micro-lens array (Fig. 1(c)). The interpolated surface phase profile obtained by using the light spot distribution calculated derivatives, is presented in Fig. 4(a) with the unit of radian. The surface predicts an obvious quadratic inhomogeneity linked with the mechanical stress employed during the fabrication, with the peak-to-valley (PV) surface profile error of 28.01 radians.

To eliminate this surface phase profile error, the quadratic shape of that in Fig. 4(a) should be compensated. Under this circumstance, we send the inversed phase distribution that depicting Fig. 4(a) to the LCoS display to compensate the original surface aberration. The LCoS display with the modified phase distribution addressed is tested with the same principle (Shack-Hartmann method) for the second time. At this moment, the light spot is uniformly distributed and the surface profile after the integration is nearly flat. The compensated surface profile is shown in Fig. 4(b). Compared to Fig. 4(a), Fig. 4(b) describes a significant improvement in the flatness, with the surface PV error decreased from 28.01 radians to only 1.28 radians. This surface calibration, based on a Shack-Hartmann method, represents its usefulness in the microparticle manipulation as it provides a more accuracy phase distribution in the generation of the light bottle structure for optical trapping.
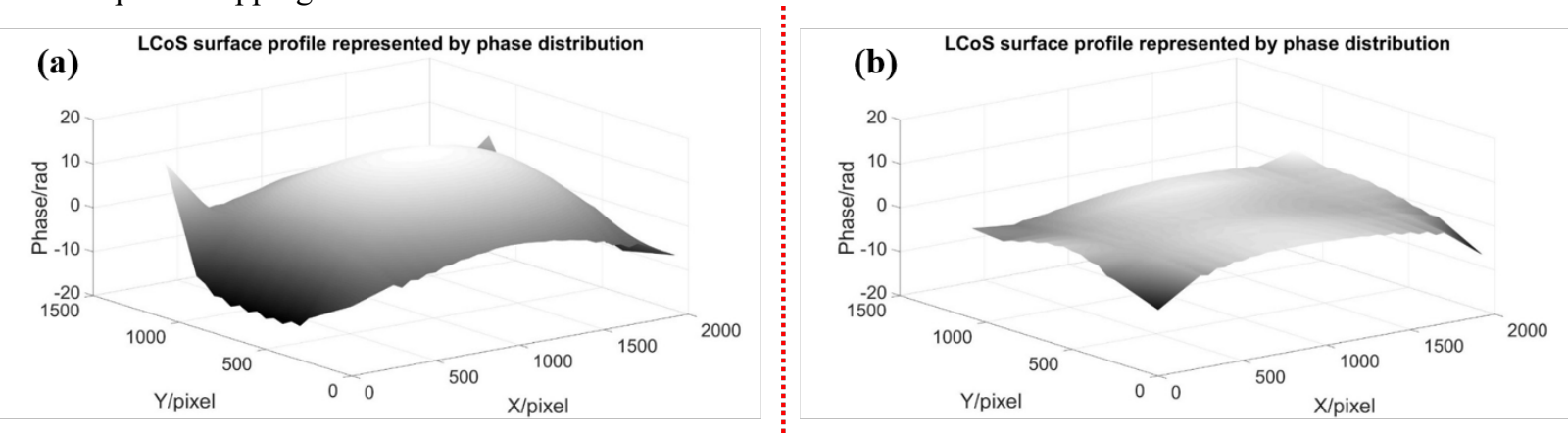

Fig. 4: (a) LCoS surface phase profile retrieved from the Shack-Hartmann method before the calibration; (b) LCoS surface phase profile retrieved after the modification.

\section{MICROPARTICLE MANUPULATION BY USING A CALIBRATED LCOS DISPLAY}

In this section, we mainly discuss the usage of the optimized LCoS display realized in Section 2 to achieve the microparticle manipulation with optical bottle structures generated by different dynamic split-lens configurations. In particular, we introduce the photophoretic force [16] to firstly trap the particle in the air and then control the position of the particle to perform the spatial dragging. The light structures created by the spilt-lens configurations is heating the environment which increase the molecular energy of the gas passing through this bottle structure. This optical-excited thermal force is described as photophoretic force. Hence, the gravity of one small particle dropped into this bottle is compensated by the photophoretic force, this leads to a statistic particle trapping. The schematic figure of the optical particle trapping is shown in Fig. 5, where a circle microsphere is flowing in the air circled by the optical bottle. Moreover, any change of the spatial location of this force will bring about the displacement to the particle, which as 
depicted before, forces the particle to be dragged. However, it is difficult for a particle to drop into the light bottle structures because the photophoretic force formed by the upper section of the bottle prohibits the entering of the particle into the bottle structure. Therefore, an optical-excited bottle structure with the upper section opened is preferable for the particle to directly drop into the trap. Afterwards, in order to realize the optical dragging, this already opened upper section in the bottle structure should be closed. In this case, a reconfigurable bottle structure which is able to adjust its spatial shape is mandatory. Thanks to the capability of the LCoS display, an income light can be shaped into any favorable shape with certain phase distributions driven. In our case, we use the SLM generated split-lens configurations to form any reconfigurable light bottle structures, feasible to open or close the upper section of the bottle light structure, to manipulate the microparticles.

To obtain a certain split-lens configuration, a lens is to be split into several sections and we separate these different sections for a distance. In this case, we obtain numbers of small sectorial lenses with each sectorial lens has their corresponding focal length. Afterwards, the income light illuminating each split-lens is focused into their corresponding focal points and a light spot distribution is obtained. Note that the number of the spots is equal to the sectors of the splitlenses and their spatial positions correspond to the distribution of different sectors. If the light passing through each splitlens is continuously propagating into the further space after the focal plane, they meet each other and the interference is to be presented if the illumination light is coherent. One classic example of the split-lens is the Billet-lens configuration above-mentioned generating an interference pattern for the phase-voltage calibration. Moreover, the split-lens configurations similar to the Billet-lens scheme but provide more sectors are also of great interest to form more interference patterns. For instance, we can provide a continuous split-lens scheme containing $\mathrm{N}$ sectorial split-lens with any lens shares a specific distance to the lens center. The light spot pattern generated on the focal plane is a circle ring and the interference pattern obtained in the further space presents a Poisson distributed pattern.

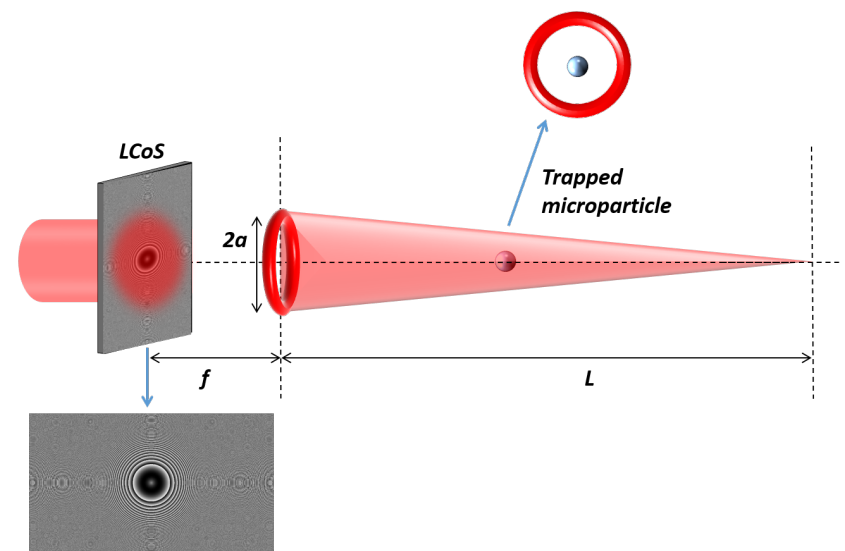

Fig. 5: The light cone structure realized by introducing a split-lens configuration to achieve the microparticle trapping.

Under this scenario, different three-dimensional optics structures can be easily achieved by simple differing the split-lens structures and their spatial distributions. However, two major problems arise during the utilizing of the splitlens configurations demonstrated as the difficulty in physically fabricating a precise split-lens and spatially calibrate each sector into their deserved positions. Hence, we use an LCoS display, feasible to generate different DOEs, to obtain the digital generated split-lens configurations. Note that this not only ease the dynamic generation of the split-lens configuration for the particle manipulation, but also avoid the stray light passing through the slits in a physical lens based split-lens.

\section{A. Split-lens configuration based light structures for the microparticle manipulation}

To realize the optical trapping, a particular three-dimensional light cone structure generated by the LCoS display using the continuous split-lens configuration is utilized. The phase distribution of this continuous split-lens scheme is detailed in Eq. 1.

$$
U(r, \theta)=\exp \left[\frac{i \pi}{f \lambda}(r-a(\theta))^{2}\right]
$$

Note that Eq. 1 is presented in the polar coordinate system where $r$ and $\theta$ are located in the SLM plane. What is more, $a(\theta)$ represents the distance defined from the split-lens sector to the coordinate origin depending on the polar angel, $\lambda$ for the wavelength of the illumination implemented to the LCoS display and $f$ stands for the focal length of the 
continuous split-lens. Note that a symmetric light cone structure is preferable to trap the particle because it provides a uniformly distributed photophoretic force, hence we set the parameter $a(\theta)$ as a constant no longer depends on the polar angel $\theta$. By setting properly the focal length and the split-lens separated distance, the implemented phase distribution modulates the income illumination into a cone structure established at a certain propagated plane $S$ is demonstrated as Fig. 5. The aperture of the light cone at each spatial position is gradually decreased from the largest diameter to a focused point. The length of the light cone can be represented as Eq. 2,

$$
L=\frac{a f}{\phi}
$$

where $a$ refers to the separation distance of the split-lens on the LCoS display and $f$, as above-mentioned, means the focal length of the split-lens configuration. On the other hand, $\phi$ is denotes as the whole aperture of the lens configuration without splitting. At last, we want to mention that the tail end of the cone structure in fact is not a point but a diffusion spot considering the diffraction of the split-lens pattern on the LCoS display.

Note that the light cone structure in Fig. 5 is closed on the upper part, indicating the photophoretic force formed by the air heated by this particular section restricts the particle from dropping into the inner hollow area. Therefore, we set up a "sunroof" structure on the top of the whole light cone structure to realize the microparticle dropping. This is achieved by removing the phase distribution corresponds to this upper section in the LCoS display by multiplexing a black triangle, forming a whole phase scheme represented in Fig. 6. In this case, the whole upper section of the light cone structure shown by Fig. 5 is deprived, leaving the light cone for particle trapping represented as Fig. 6.

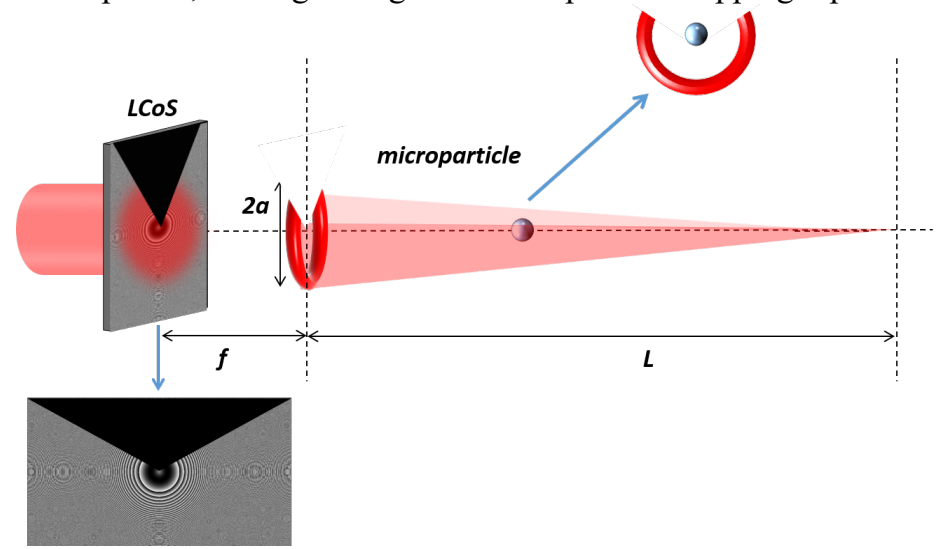

Fig. 6: The light cone structure with the upper section opened to ease the particle from dropping by introducing a black triangle shape zero-phase distribution in the LCoS display.

Once the particle is dropped from the upper section into the light cone from the opening region, the cone needs to be closed in order to fully trap the particle inside. As the light cone is opened by the introduction of a zero-phase section to the upper region on the LCoS display, we can easily achieve the light structure closing simply by removing the same black triangle to the similar section. The light cone scheme now after the closing shares the equivalent shape as it of Fig. 5.

At this moment, the microparticle is stably trapped in the hollow area of the light cone structure. However, we notice that the cone structure only guarantees one side (the tail side) of the whole scheme to be closed but the front side is not sealed. Therefore, the trapped particle cannot be spatially manipulated since the displacement of the light cone deteriorates the particle to a situation that it is not located in the hollow area any more. Under this scenario, a second regular diffractive lens with its focal plane located at a same place as the front ring side of the first light cone structure, is introduced to seal the opening section to form an integral light cone. To obtain the original and the reversed light cone structures from the LCoS display, the phase distribution of the second regular diffractive lens is multiplexed to the SLM with its focal length smaller than the original one. The final sealed light cone structure is demonstrated as the cross section (highlighted by the dark red lines) in Fig. 7. 


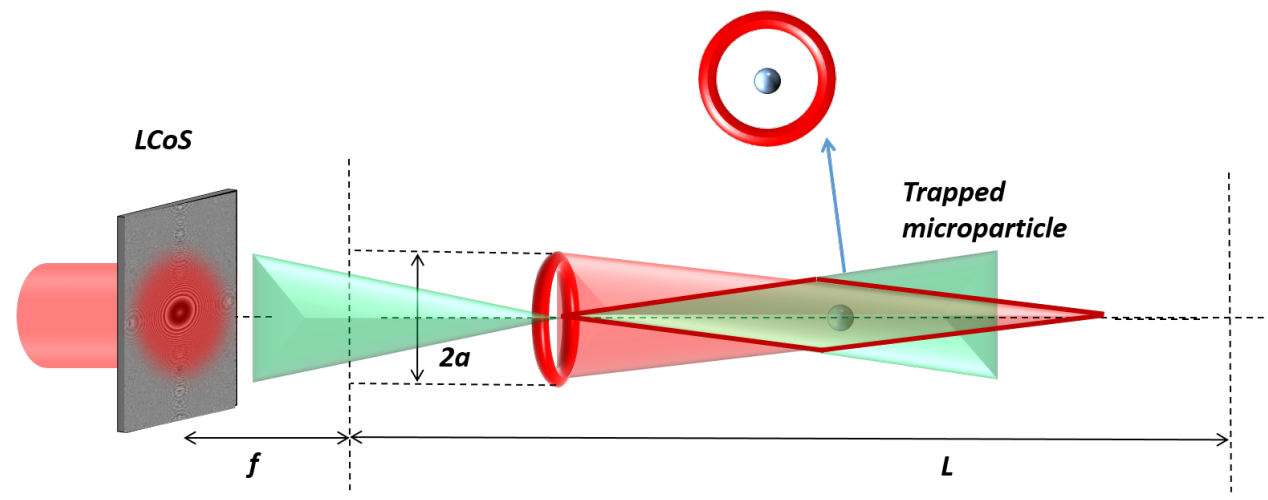

Fig. 7: The sealed light cone structure generated by multiplexing a second regular diffractive lens to the LCoS display.

Once the particle is steadily trapped, the particle can be displaced by changing the spatial position of the light capsule structure. Specifically, the photophoretic force arisen by the optical characters of the optical bottle, maintaining the particle to be suspended in the air, is not adjusted except for its physical location if any light cone displacement is introduced. Therefore, by simply digitally modifying the parameters of the LCoS generated split-lens configurations (i.e., the focal length $f$ or the separation distance $a$ ) applied to form the light cones, the particle manipulation is available.

\section{B. Experimental implementation for the microparticle manipulation}

To experimentally verify the feasibility of particle trapping with LCoS display driven split-lens configurations, we experimentally implement the optical scheme discussed above. The experimental set-up is presented in Fig. 8.

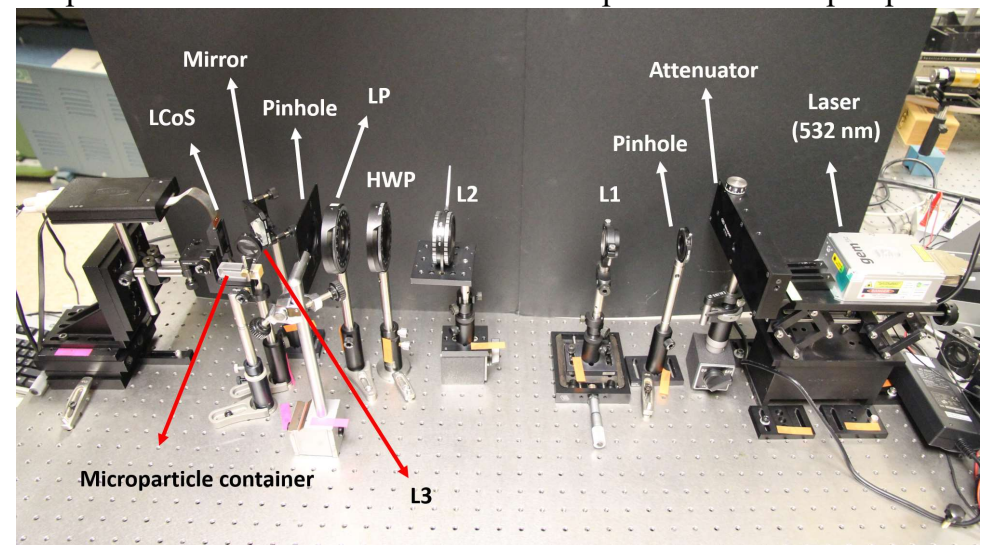

Fig. 8: Experimental set-up for the microparticle trapping.

A $500 \mathrm{~mW}$ semiconductor laser with the central wavelength of $532 \mathrm{~nm}$ distributed by Laser Quantum. Co (gem 532 Model) is applied as the illumination source. Afterwards, the light passing through the attenuator, inserted to control the input light power, is filtered by the pinhole located before lens L1. Note that the divergent lens L1 and the convergent lens L2 shown in Fig. 8 form an afocal optical system which both collimates the input light and expands the size of the laser light spot. The beam spot size after the expansion presents a diameter of $3.38 \mathrm{~mm}^{2}$. The collimated light then is modified to linear polarized with the half waveplate and the implanted linear polarizer oriented to a certain direction. Moreover, the intensity of the light illuminating the follow-up optical system, is easily adapted to the optimal condition by rotating the half waveplate. At this moment, the linear polarized light is again filtered by the second pinhole in order to better illuminate the LCoS display. Note that the LCoS display is the critical element in the whole system set-up because the light cone structures are realized by different diffractive optical elements (DOEs) digitally formed on the LCoS. In our case, we are using a parallel aligned reflective LCoS display distributed by HOLOEYE with the resolution of $1920 \times 1080$ pixels. The filling factor of this SLM is $87 \%$ and the pixel size is of $8 \mu \mathrm{m}$. This device is able to achieve a total light efficiency of more than $50 \%$ because it provides a reflectivity of $60 \%$ to $75 \%$ (depends on the model) and a diffraction efficiency of more than $80 \%$. Hence, by addressing particular DOEs to this liquid crystal device, the light cones discussed in the previous section are obtained at the certain spatial locations. However, the LCoS display is a reflective device, implying the light bottle structures are to be generated in the reflection interval which is hardly to be applied. To solve this problem, we calibrate the LCoS display with its incident angle deviates from the optical axis a small value of 2 degrees. In other words, the LCoS plane is not precisely perpendicular to the optical axis, but the normal 
of the LCoS surface is slightly tilted from the optical axis for a little angel. Once the reflected image is tilted, a flat mirror is implemented in a proper position to steer the images modulated by the LCoS to another optical arm vertical to the former optical axis. In our case, the desired light cone structures are reflected to the axis which is determined by the mirror and the center of the convergent lens L3. The purpose to introduce this third lens is to compact the threedimensional bottle structures to a certain length to ease the optical trapping. Finally, a transparent microparticle container is placed at the fixed location in a way the light cone structures are acquired. The particles injected into the container are carbon coated glass microspheres with the diameters ranging from $60 \mu \mathrm{m}$ to $75 \mu \mathrm{m}$. These microspheres, adhered to the inner surfaces of the container by electrostatic force, is easily segregated from the surface with insignificant force such as the force fulfilled by softly tapping the surface. Under this scenario, the surface detached particles in the container are trapped in the three-dimensional light bottle structures located in the place where the container stands.

Once the experimental set-up is completely implemented, different phase distributions representing the DOEs are addressed to the LCoS. We firstly generate a light bottle structure with the front size opened (i.e., Fig. 5) to verify the usefulness of the proposed method. Specifically, the separation distance $a$ is set as $1.07 \mathrm{~mm}$, the focal length $f$ as $370 \mathrm{~mm}$ and the whole lens aperture $\phi$ as $2.07 \mathrm{~mm}$. Hence, the light cone length is calculated as $5.52 \mathrm{~mm}$ considering the size compaction provided by L3 lens. Meanwhile, a CCD camera located on a linear displacement stage is implemented after the L3 lens to measure the cone shape and the length. The measured light cone shapes at the front side and the tail side are demonstrated as Figs. 9(a) and (b), respectively. The spatial distance ranging from the Fig. 9(a) scheme location to the place where Fig. 9(b) scheme appears is $6.5 \mathrm{~mm}$, agree with the theoretically calculated $5.52 \mathrm{~mm}$ because the light cone shows a focal depth of $\sim 0.5 \mathrm{~mm}$ on both the front side and the tail side (this means the total focal depth error is 1 mm). Note that Fig. 9(a) demonstrates a light spot in the center and Fig. 9(b) presents a diffused spot instead of a light spot owing to the diffraction of the LCoS display that introduce diffraction of the LCoS dislay.
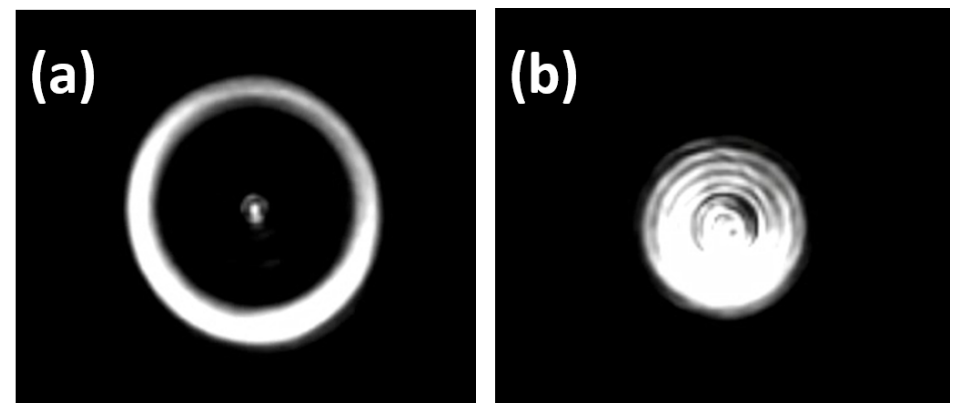

Fig. 9: Cross section of the light cone experimentally obtained by addressing the split-lens configuration: (a) the front side cross section, and (b) the tail side cross section.

The light cone obtained is later reshaped by opening the upper section to ease the particle to be dropped into the hollow section. In the experimental implementation, nothing but only the phase distribution addressed to the LCoS display is changed by multiplexing a black triangle (the top angle of the isosceles triangle as 120 degrees) showed in Fig. 6 to the corresponding section. Under this scenario, we use the same CCD camera to measure the light cone sketch. The light cone shapes with upper section opened at the front side and the tail side are shown in Figs. 10(a) and (b), respectively. Moreover, not only the diameters of the light rings and the light spots in Figs. 9 and 10 are the same, but also the light cone shown in Fig. 10 shares an equivalent length as the cone length of Fig. 9.
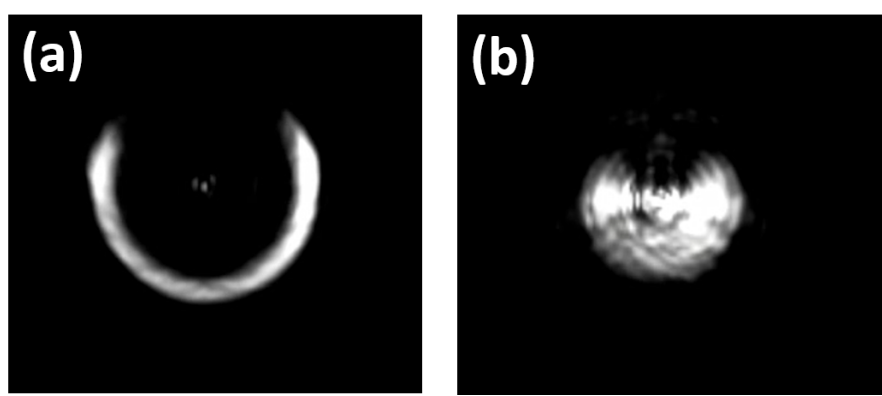

Fig. 10: Cross section of the light cone with the upper section opened experimentally obtained by addressing the black triangle pattern to the original split-lens configuration: (a) the front side cross section, and (b) the tail side cross section.

Meanwhile, the particle drop into the light bottle structure as Fig. 10(a) from above is suspended in the hollow area. However, to stably trap the particle in the bottle structure, the upper opening section should be covered and the front side 
should be seized. As above theoretically discussed, it is easily achievable to close the upper section only by removing the black triangle on the phase distribution. What is more, by simply introducing another diffractive lens with a shorter focal length than it of the first light cone structure to the LCoS by pixel multiplex, the front side is closed as well. In particular, the focal length of the second diffractive lens is chosen as $350 \mathrm{~mm}$ (the focal length of the original light cone is of 370 $\mathrm{mm}$ ). The figures of the capsule shaped light cone (i.e., dark red structure in Fig. 7) observed from the front plane, the middle plane and the tail plane are presented in Figs. 11(a), (b) and (c), respectively. It is obvious demonstrated that both side of the cone structure is seized and the light cone gives a length of $6.5 \mathrm{~mm}$ as well.
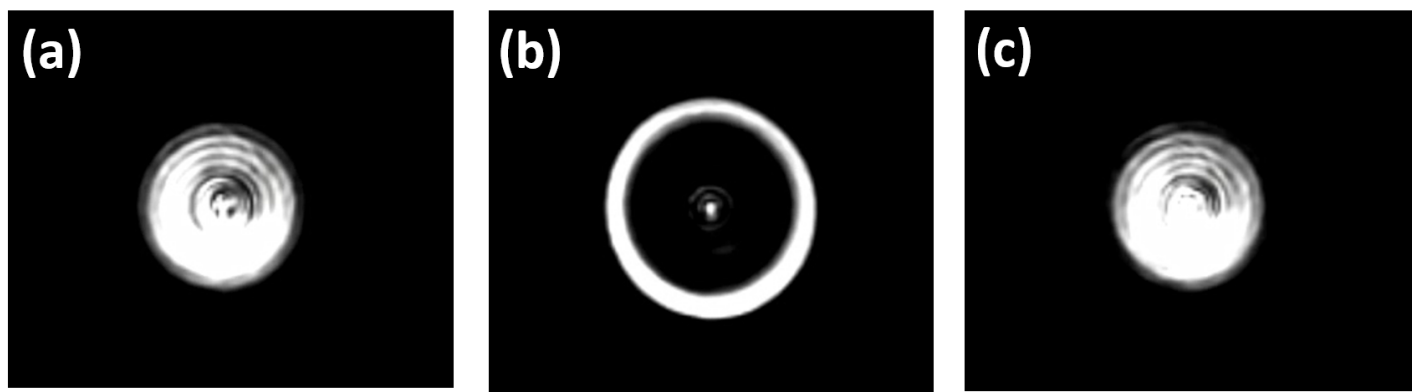

Fig. 11: Cross section of the light capsule structure experimentally obtained by closing the upper side and the front side: (a) the front side cross section, (b) the middle cross section; and (c) the tail side cross section.

What is more, we also steadily captured one microparticle trapped in the capsule structure signified by Fig. 11 . The trapped particle is given in Fig. 12(a).

Apart from the particle trapping, the particle manipulation is also demonstrated within the experiment. Specifically, the focal length of the original split-lens configuration is changed from $370 \mathrm{~mm}$ to $380 \mathrm{~mm}$, the focal length of the multiplexed second diffractive lens is changed from $350 \mathrm{~mm}$ to $361 \mathrm{~mm}$. In this case, the spatial positon of the new generated light cone is displaced to a new specific location, offering an axial dragging of the whole capsule structure along the optical axis for a distance of nearly $0.65 \mathrm{~mm}$, fitting the theoretical calculation of $0.47 \mathrm{~mm}$. Therefore, the particle trapped in the light capsule is also axially dragged for a certain distance. The trapped particle after the axial dragging is shown in Fig. 12(b). Compare the spatial locations of Fig. 12(a) to that of Fig. 12(b), the spatial displacement of the microparticle located in the container is easily distinguishable.
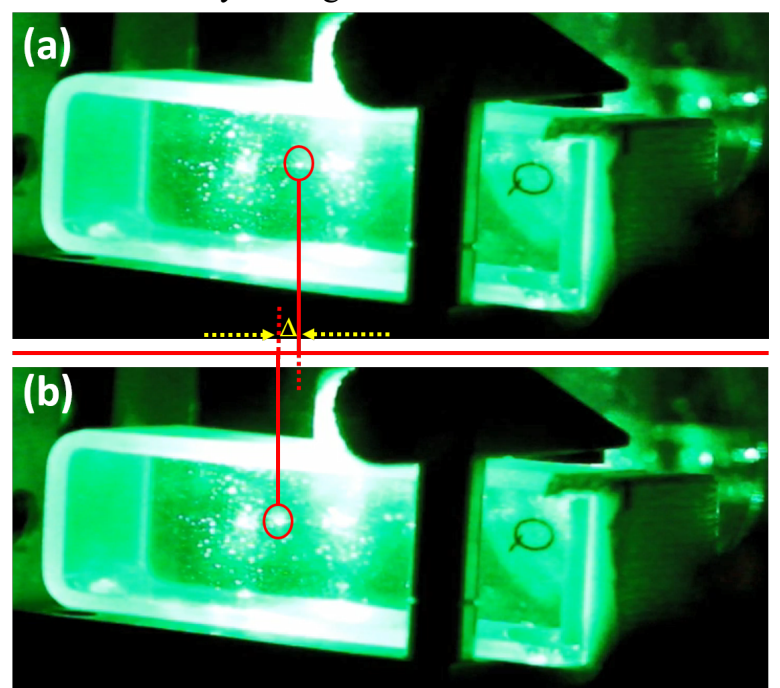

Fig. 12: Trapped microparticle in the container in different spatial positions: (a) the trapped microparticle before the spatial dragging, and (b) the trapped microparticle after the spatial dragging.

\section{CONCLUSIONS}

In this paper we proposed a microparticle manipulation method based on a self-calibrated Liquid Crystal on Silicon (LCoS) display by using dynamic light-structures. Specifically, two main contributions are proposed and discussed, respectively. On the one hand, we firstly achieved the LCoS display self-calibration by implementing two self-generated diffractive optical elements (DOEs) to the LCoS described as the Billet-lens configuration and the micro-lens array 
pattern (Shack-Hartmann pattern). The addressed Billet-lens configuration is suitable to generate an interference fringe from which the phase-voltage curve of the studied device is obtained. The micro-lens array, feasible to generate the focal spot array from which the wavefront aberration is revealed, determines the LCoS surface phase profile. Afterwards, we implied the calibrated results to the LCoS display to optimize its performance for particle manipulation. On the other hand, the particle manipulation is realized with the same but optimized LCoS display by addressing different split-lens configurations feasible to form three-dimensional light cone structures. By digitally control the configurable light structures, the particle is stably trapped into the hollow area of a light capsule structure and spatially displaced by easily modifying some control parameters of the split-lens configurations.

The capability of the proposed methods is experimentally verified by implementing the LCoS self-calibration and the microparticle manipulation, respectively. Above all, the LCoS calibration is firstly implemented because the particle trapping is to be realized with an optimized device. In this case, we achieved a linear tendency phase-voltage curve ranging from 0 to $\sim 6.28$ radians with a $0.4 \mathrm{~mm}$ separated Billet-lens configuration. Moreover, the quadratic LCoS surface profile is modified to a flat uniform distribution with a $32 \times 16$ distributed spot array. Afterwards, a carbon coated microsphere as the diameter of 60-75 $\mu \mathrm{m}$, is trapped with the optimized LCoS by the generation of a $6.5 \mathrm{~mm}$ long optical capsule structure. Finally, the trapped particle is spatially dragged for a small distance by digitally changing the split-lens configuration for $11 \mathrm{~mm}$.

\section{REFERENCES}

[1] Prentis, M., Cable, A., Bjorkholm. J.E., Chu, S., Raab, E.L., and Pritchard, D.E., "Atomic-density-dependent losses in an optical trap," Opt. Lett, 13(6), 452-454 (1988).

[2] Ashkin, A., "Optical trapping and manipulation of neutral particles using lasers," Proc. Natl. Acad. Sci. U.S.A., 94(10) 4853-4860 (1997).

[3] Miesner, H.J., Grimm, R., Grieser, M., Habs, D., Schwalm, B., and Wolf, A., "Efficient, indirect transverse laser cooling of a fast stored ion beam," Phys. Rev. Lett, 77(4), 623 (1996).

[4] Kasevich, M., and Chu, S., "Atomic interferometry using stimulated Raman transitions," Phys. Rev. Lett, 67(2), 181 (1991).

[5] Aminoff, C.G., Steane, A.M., Bouyer, P., Desbiolles, P., Dalibard, J., and Cohen-Tannoudji, C., "Cesium atoms bouncing in a stable gravitational cavity," Phys. Rev. Lett, 71(19), 3083 (1993).

[6] Renn, M.J., Montgomery, D., Vdovin, O., Anderson, D.Z., Wieman, C.E., and Cornell, E.A., "Laser-guided atoms in hollow-core optical fibers," Phys. Rev. Lett, 75(18), 3083 (1995).

[7] Ashkin, A., and Dziedzic, J.M., "Optical trapping and manipulation of viruses and bacteria," Science, 235(4795), 1517-1520 (1987).

[8] Ashkin, A., Dzeidzic J.M., and Yamane, T., "Optical trapping and manipulation of single cells using infrared laser beams," Nature, 330(6150), 769 (1987).

[9] Liang, H., Wright, W.H., Cheng, S., He, W., and Berns, W.W., "Micromanipulation of Chromosomes in PTK2 Cells Using Laser Microsurgery (Optical Scalpel) in Combination with Laser-Induced Optical Force (Optical Tweezers)," Exp. Cell Res, 204(1), 110-120 (1993).

[10]Desyatnikov, A.S., Shvedov, V.G., Rode, A.V., Krolikowski, W., and Kivshar, Y.S., "Photophoretic manipulation of absorbing aerosol particles with vortex beams: theory versus experiment," Opt. Express, 17(10), 8201-8211 (2009).

[11] Shvedov, V.G., Rode, A.V., Izdebskaya, Y.V., Desyatnikov, A.S., Krolikowski, W., and Kivshar, Y.S., "Giant optical manipulation," Phys. Rev. Lett, 105(11), 118103 (2010).

[12] Shvedov, V.G., Hnatovsky, C., Rode, A.V., and Krolikowski, W., "Robust trapping and manipulation of airborne particles with a bottle beam," Opt. Express, 19(18), 17350-17356 (2011).

[13] Shvedov, V., Davoyan, A.R., Hnatovsky, C., Engheta, N. and Krolikowski, W., "A long-range polarizationcontrolled optical tractor beam," Nat. Photon, 8(11), 846-850 (2014).

[14] Eckerskorn, N., Bowman, R., Kirian, R.A., Awel, S., Wiedorn, M., Kupper, J., Padgett, M.J., Chapman, H.N., and Rode, A.V., "Optically induced forces imposed in an optical funnel on a stream of particles in air or vacuum," Phys. Rev. Appl, 4(6), 064001 (2015).

[15] Kovalev, A.A., Kotlyar, V.V. and Porfirev, A.P., "Optical trapping and moving of microparticles by using asymmetrical laguerre-gaussian beams," Opt. Lett, 41(11), 2426-2429 (2016). 
[16] Jovanovic, O. "Photophoresis: Light induced motion of particles suspended in gas," J Quant Spectrosc Radiat Transf, 110(10), $889-901$ (2009).

[17]Lizana, A., Vargas, A., Turpin, A., Ramírez, C., Estevez, I., and Campos, J., "Shaping light with split lens configurations," J Opt, 18(10), 105605 (2016).

[18]Zhang, Z., You, Z., and Chu, D., "Fundamentals of phase-only liquid crystal on silicon (LCoS) devices," Light Sci Appl, 3(10), e213 (2014).

[19] Moreno, I., Lizana, A., Campos, J., Márquez, A., Iemmi, C., and Yzuel, M.J. "Mueller-Stokes characterization and optimization of liquid crystal on silicon display showing depo- plarization," Opt Express 16(3), 1669-1685 2008.

[20]Zhang, H., Liu, H., Lizana, A., Xu, W., Campos, J., and Lu, Z., "Methods for the performance enhancement and the error characterization of large diameter ground-based diffractive telescope," Opt. Express, 25(22), 2666226677 (2017).

[21] Mu, Q., Cao, Z., Hu, L., Li, D., and Xuan, L., "Adaptive optics imaging system based on a high resolution liquid crystal on silicon device," Opt. Express, 14(18), 8013-8018 (2006).

[22] Bowman, R.W., Wright, A.J., and Padgett, M.J., "An SLM-based Shack-Hartmann wavefront sensor for aberration correction in optical tweezers," J Opt, 12(12), 124004 (2010).

[23] Lizana, A., Márquez, A., Lobato, L., Rodagne, Y., Moreno, I., Iemmi, C., and Campos, J., "The minimum Euclidean distance principle applied to improve the modulation diffraction efficiency in digitally controlled spatial light modulators," Opt Express, 18(10), 10581-10593 (2010).

[24] Márquez, A., Moreno, I., Iemmi, C., Lizana, A., Campos, J., and Yzuel, M.J., "Mueller-Stokes characterization and optimization of a liquid crystal on silicon display showing depolarization," Opt Express, 16(3), 1669-1685 (2008).

[25]Lizana, A., Moreno, I., Iemmi, C., Márquez, A., Campos, J., and Yzuel, M.J., "Time-resolved Mueller matrix analysis of a liquid crystal on silicon display," Appl Opt, 47(23), 4267-4274 (2008).

[26] Rodrigo. J., and Alieva. T., "Freestyle 3D laser traps: tools for studying light-driven particle dynamics and beyond," Optica, 2(9), 812-815 (2015).

[27]Zhang. H., Lizana. A., Iemmi. C., Monroy-Ramírez, F.A., Márquez. A., Moreno. I., and Campos. J., "LCoS display phase self-calibration method based on diffractive lens schemes," Opt Lasers Eng, 106, 147-154 (2018).

[28] Vargas, J., Uribe-Patarroyo, N., Quiroga, J.A., Alvarez-Herrero, A., and Belenguer, T., "Optical inspection of liquid crystal variable retarder inhomogeneities," Appl Opt, 49(4), 568-574 (2010).

[29] Martínez, J.L., Fernández, E.J., Prieto, P.M., and Artal, P., "Interferometric method for phase calibration in liquid crystal spatial light modulator using a self-generated diffraction-grating," Opt Express, 24(13), 1415914171 (2017).

[30] Ramirez, C., Karakus, B., Lizana, A., and Campos, J., "Polarimetric method for liquid crystal displays characterization in presence of phase fluctuations," Opt Express, 21(3), 3182-3192 (2013).

[31]Bergeron, A., Gauvin, J., Gagnon, F., Gingras, D., Arsenault, H.H., and Doucet, M., "Phase calibration and applications of a liquid crystal spatial light modulator," Appl Opt, 34(23), 5133-5139 (1995).

[32] Engström, D., Milewski, G., Bengtsson, J., and Galt, S., "Diffraction-based determination of the phase modulation for general spatial light modulators," Appl Opt, 45(28), 7195-7204 (2006).

[33]Zhang, Z., Lu, G., and Francis, T.S., "Simple method for measuring phase modulation in liquid crystal televisions," Opt Eng, 33(9), 3018-3022 (1994).

[34] Schiegerling, J., and Dehoog, E., "Problems testing diffractive intraocular lenses with Shack-Hartmann sensors," Appl Opt, 47(25), 4548-4554 (2008).

[35]Lobato, L., Márquez, A., Lizana, A., Moreno, I., and Campos, J., "Characterization of a Parallel Aligned Liquid Crystal on Silicon and its application on a Shack-Hartmann sensor," Proc. SPIE 7797, 77970Q-1 (2010). 